\title{
Most primary adrenal lymphomas are diffuse large B-cell lymphomas with non-germinal center B-cell phenotype, BCL6 gene rearrangement and poor prognosis
}

\author{
Ana Mozos ${ }^{1}$, Hongtao $\mathrm{Ye}^{2}$, Wen-Yu Chuang ${ }^{3}$, Jan-Show Chu ${ }^{4}$, Wan-Ting Huang ${ }^{5}$, \\ Han-Ku Chen ${ }^{5}$, Yung-Hsiang Hsu ${ }^{6}$, Chris M Bacon ${ }^{7}$, Ming-Qing Du ${ }^{8}$, Elias Campo ${ }^{1}$ \\ and Shih-Sung Chuang ${ }^{4,9}$
}

${ }^{1}$ Department of Anatomic Pathology, Hospital Clinic, University of Barcelona, Barcelona, Spain; ${ }^{2}$ Department of Histopathology, Royal National Orthopaedic Hospital NHS Trust, University College London, London, UK; ${ }^{3}$ Department of Pathology, Chang Gung Memorial Hospital and Chang Gung University School of Medicine, Taoyuan, Taiwan; ${ }^{4}$ Department of Pathology, Taipei Medical University, Taipei, Taiwan; ${ }^{5}$ Department of Pathology, Chang Gung Memorial Hospital-Kaohsiung Medical Center and Chang Gung University College of Medicine, Taoyuan, Taiwan; ${ }^{6}$ Department of Pathology, Buddhist Tzu Chi Medical Center and University, Hualien, Taiwan; ${ }^{7}$ Northern Institute for Cancer Research, Newcastle University, Newcastle upon Tyne, UK; ${ }^{8}$ Department of Pathology, University of Cambridge, Cambridge, UK and ${ }^{9}$ Department of Pathology, Chi-Mei Medical Center, Tainan, Taiwan

\begin{abstract}
Primary adrenal lymphoma is extremely rare, accounting for $<1 \%$ of non-Hodgkin lymphomas, and lymphomaassociated chromosomal translocations have yet to be reported in this entity. We performed a retrospective study of 10 cases in immunocompetent patients including 4 males and 6 females with a median age of 68 years. The most common presenting symptoms were abdominal pain and fever; unexpectedly, clinically evident adrenal insufficiency was detected only in one patient. The mean tumor size at diagnosis was $8.5 \mathrm{~cm}$. Half of the patients had bilateral involvement. All cases presented with stage IE disease without regional nodal involvement. Histologically, eight cases were diffuse large B-cell lymphoma, all of which carried a nongerminal center B-cell phenotype. Fluorescence in situ hybridization revealed BCL6 gene rearrangement in 5 $(83 \%)$ of 6 diffuse large B-cell lymphomas investigated. The remaining cases were one case each of plasmablastic lymphoma and extranodal NK/T-cell lymphoma, nasal type, the first and third case of primary adrenal lymphoma of these particular lymphoma subtypes in the English literature, respectively. At a median follow-up of 4.5 months, 7 patients died of lymphoma, 1 died of an unrelated disease, 1 was alive with disease, and 1 was alive without disease. The prognosis of these patients was poor as compared with those with nodal diffuse large B-cell lymphoma. We speculate that the poor outcome of primary adrenal lymphoma might be related to the bulky tumor size at presentation, non-germinal center B-cell phenotype, and frequent $B C L-6$ gene rearrangement.
\end{abstract}

Modern Pathology (2009) 22, 1210-1217; doi:10.1038/modpathol.2009.87; published online 12 June 2009

Keywords: adrenal insufficiency; diffuse large B-cell lymphoma; NK/T-cell lymphoma; plasmablastic lymphoma; primary adrenal lymphoma; Taiwan

Although secondary involvement of the adrenal glands by non-Hodgkin's lymphoma is not uncom-

Correspondence: Dr S-S Chuang, MD, Department of Pathology, Chi-Mei Medical Center and Taipei Medical University, 901 Chung-Hwa Road, Yung-Kang City, Tainan County 710, Taiwan. E-mail: cmh5301@mail.chimei.org.tw

Received 07 April 2009; revised 07 May 2009; accepted 08 May 2009; published online 12 June 2009 mon, primary adrenal lymphoma is extremely rare and accounts for $<1 \%$ of all non-Hodgkin's lymphoma cases. ${ }^{1}$ In the English literature, most of the papers on primary adrenal lymphomas are singlecase reports and literature reviews based on a small number of cases except two large series. ${ }^{1-5}$ Up to half of the small number of reported patients with primary adrenal lymphoma were associated with adrenal insufficiency and there is a high rate of 
bilateral adrenal involvement. ${ }^{1-5}$ However, these data might be publication bias due to the rarity of this tumor. Pathologically, the great majority of cases were diffuse large B-cell lymphomas, ${ }^{2,3}$ and when immunophenotyping were performed, these tumors usually expressed bcl-2 with a non-germinal center B-cell phenotype. ${ }^{6}$ To date, lymphoma-associated chromosomal translocations have not been reported in primary adrenal lymphoma. We conducted this retrospective study to better define the clinicopathological and molecular features of this entity. We found that most tumors were diffuse large B-cell lymphomas of non-germinal center B-cell phenotype with $B C L 6$ gene rearrangement and poor prognosis. In addition, we presented the clinicopathological findings of the first case of plasmablastic lymphoma and the third case of extranodal NK/T-cell lymphoma, nasal type arising primarily in the adrenal gland in the English literature.

\section{Materials and methods}

\section{Materials}

A retrospective search of primary adrenal lymphoma in the authors' institutions constituted this study. Only cases of primary adrenal origin with or without regional lymph node involvement were included. Those cases with secondary adrenal involvement or those occurring in HIV-infected patients were excluded.

\section{Histopathology and Immunohistochemistry}

The surgical specimens were fixed in buffered formalin and embedded in paraffin, and sections of $4 \mu \mathrm{m}$ thick were stained with hematoxylin and eosin. Immunohistochemical staining was performed using an autostainer and an antigen-retrieval technique was applied as needed for each specific antibody. The antibodies used were CD3, CD20, bcl2, bcl-6, IRF4/MUM1 (Dako Corp, Carpinteria, CA, USA), CD10 (Novocastra, Newcastle upon Tyne, UK), and cyclin D1 (LAB Vision Co, Fremont, CA, USA). Additional antibodies including epithelial membrane antigen (EMA), latency membrane protein-1 (LMP-1) of Epstein-Barr virus (EBV), CD30, CD45, CD79a, IgD and IgM (Dako), CD56, CD138 and latency-associated nuclear antigen of HHV8 (HHV8LANA; Novocastra), IgA and IgG (Zymed, South San Francisco, CA, USA) were added for Case 1. An additional panel of T-cell antibodies including CD2, CD4, CD5, CD7, CD8, CD30, CD56, (Dako Corp), TIA1 (Coulter-Immunotech, Marseille, France), Granzyme B (Monosan, San Francisco, CA), $\beta$ F1 (Endogen/Pierce Biotechnology, Rockford, IL, USA), and CD16 (Serotec, Raleigh, NC, USA) was added for Case 10.

\section{In Situ Hybridization}

In situ hybridization for EBV-encoded mRNA (EBER) was performed using an autostainer with an EBV-specific probe (Bond ISH EBER Probe, Vision BioSystems Ltd. or the INFORM EBER Probe, Ventana Medical Systems, Tucson, AZ, USA). The extent of EBER positivity was graded as: negative; $1+, 1 \%$ to $<25 \%$ cells with positive signal; $2+$, $25-49 \%$; $3+, 50-74 \%$; and $4+$, $\geqq 75 \%$.

\section{Fluorescence In Situ Hybridization}

Locus-specific interphase fluorescence in situ hybridization (FISH) was performed on $4 \mu$ thick paraffin-embedded tissue sections using $M Y C$, $I G H, B C L 2, B C L-6$, and CCND1 dual-color, breakapart rearrangement probes (Vysis/Abbott Laboratories Ltd, Maidenhead, UK) as described earlier. ${ }^{7}$ Briefly, de-paraffinized sections were pre-treated by pressure-cooking for $3 \mathrm{~min}$ in EDTA (ethylenediaminetetraacetic acid) buffer (1 mM, pH 8.0) and subsequent incubation in pepsin solution for $25 \mathrm{~min}$ at $37^{\circ} \mathrm{C}$ to increase DNA accessibility. Sections were then dehydrated through ethanol and air-dried. The appropriate probe mix $(1.0 \mu \mathrm{l})$ was applied to the tissue section and covered with a round $10 \mathrm{~mm}$ cover slip. Both probe and target DNA were simultaneously denatured at $80^{\circ} \mathrm{C}$ for $25 \mathrm{~min}$ and incubated up to 2 days at $45^{\circ} \mathrm{C}$. Post-hybridization washes were performed according to the 'rapidwash protocol' provided by Vysis, Downers Grove, IL, USA. Sections were counterstained with 4,6diamidino-2-phenylindole (DAPI) and mounted in Vectashield antifade solution (Vector Laboratories, Burlingame, CA, USA). Image acquisition and processing was performed as described earlier. ${ }^{8}$

\section{Results}

\section{Clinical Features}

A total of 10 cases of primary adrenal lymphoma were identified. The first six cases were Taiwanese, the seventh British, and the last three cases, Spanish. The patients were four males and six females, and the clinical findings are summarized in Table 1. Both the mean and the median age were 68 years (range, 48-83 years). The most common presenting symptoms were pain (usually abdominal) (6/10) and/or fever (4/10). B-symptoms (fever, weight loss of $>10 \%$ within 6 months, or night sweating) were observed in 8 out of 10 cases $(80 \%)$. Adrenal insufficiency was detected in only one patient (Case 10), although in most of the cases, no hormone study was performed due to the lack of clinical and laboratory features of adrenal insufficiency. The serum level of lactate dehydrogenase (LDH) was elevated in $7(78 \%)$ out of 9 cases tested. 


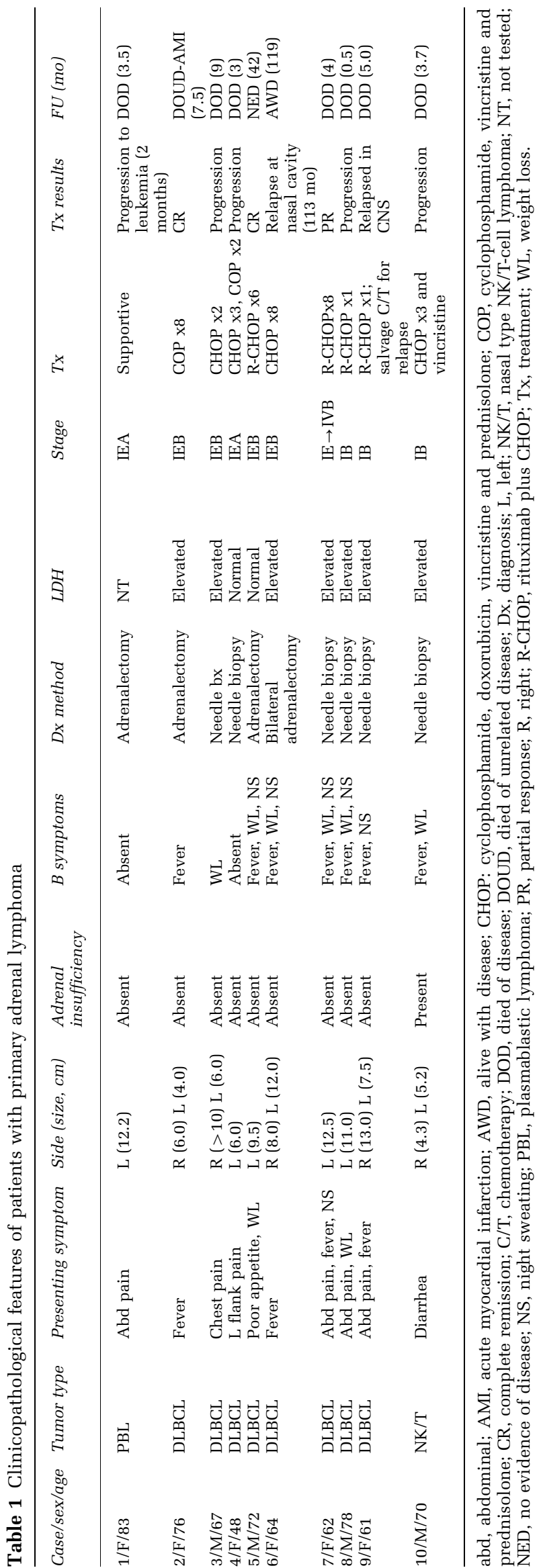

Five patients presented with unilateral involvement affecting the left adrenal gland, and the remaining five patients had bilateral adrenal involvement. The mean size of the tumor at the time of diagnosis was $8.5 \mathrm{~cm}$, with a range from 4 to $13 \mathrm{~cm}$; 6 were $>10 \mathrm{~cm}$ in diameter. The most common diagnostic procedure was needle biopsy (6/10, $60 \%)$. The remaining four patients underwent unilateral (three patients) or bilateral adrenalectomy (one patient). All patients presented with stage IE disease by the Ann-Arbor Staging System without nodal involvement. After diagnosis, nine patients received chemotherapy with a CHOP (cyclophosphamide, doxorubicin, vincristine, and prednisolone)-based regimen, some with additional rituximab. One patient received only supportive treatment. Six patients progressed despite treatment, 1 patient relapsed in the nasal cavity 113 months after the diagnosis, 1 achieved a partial response and 2 achieved complete remission. At a median follow-up of 4.5 months (mean, 19.7; range, 0.5-119 months), 7 patients died of lymphoma, 1 died of acute myocardial infarction, 1 was alive with disease, and 1 was alive with no evidence of disease.

\section{Pathologic Findings}

The first nine cases were of B-cell lineage, and the last case was an extranodal NK/T-cell lymphoma, nasal type. The immunohistochemical findings of the first nine cases are summarized in Table 2. The first case showed a diffuse, focally necrotic, monomorphic infiltration of plasmablasts with a large centrally located nucleolus and eosinophilic to amphophilic cytoplasm (Figure 1). Focal plasmacytic differentiation was discerned. The tumor cells expressed CD45, CD79a, CD138 (partial), bcl-2, IRF4/MUM1, and IgM. They were negative for CD3, CD10, CD20, CD30, CD56, bcl-6, EMA, cyclin D1, HHV8-LANA, IgA, IgD, or IgG. A fraction (5$10 \%$ ) of tumor cells were positive for LMP-1. This case was classified as plasmablastic lymphoma with plasmacytic differentiation. The remaining eight Bcell lymphomas were diffuse large B-cell lymphomas with diffuse infiltration by large atypical lymphocytes without a follicular growth pattern. Immunohistochemically, the tumors expressed CD20 (8/8), bcl-2 (8/8), bcl-6 (6/7), and IRF4/ MUM1 (7/7). All were negative for CD3 (8/8), CD10 (8/8), and cyclin D1 (7/7). The seven cases with complete immunostaining were all classified as non-germinal center B-cell phenotype based on the criteria by Hans et al. ${ }^{9}$

The last case was an extranodal NK/T lymphoma, nasal type with tumor cells expressing CD2, CD3, $\mathrm{CD} 7, \mathrm{CD} 8$, and $\beta \mathrm{F} 1$, but not CD5 (Figure 2). Furthermore, the tumor cells were positive for CD56, TIA-1, and granzyme B. Immunostaining for LMP-1 and CD30 was negative. Attempts were made to determine whether this lymphoma carried clon- 
Table 2 Results of immunophenotyping and in situ hybridization of primary adrenal lymphoma of B-cell phenotype

\begin{tabular}{|c|c|c|c|c|c|c|c|c|c|c|c|c|}
\hline \multirow[t]{2}{*}{ Case } & \multirow[t]{2}{*}{ Tumor type } & \multicolumn{5}{|c|}{ Immunohistochemistry } & \multirow[t]{2}{*}{$E B E R$} & \multicolumn{5}{|c|}{ Fluorescent in situ hybridization } \\
\hline & & CD10 & Bcl-6 & MUM1 & Cyclin D1 & Phenotype & & $C-M Y C$ & $I G H$ & BCL2 & $B C L 6$ & $C C N D 1$ \\
\hline 1 & PBL & - & - & + & - & Non-GCB & $4+$ & - & - & - & - & - \\
\hline 2 & DLBCL & - & + & + & - & Non-GCB & - & - & - & - & + & - \\
\hline 3 & DLBCL & - & - & + & - & Non-GCB & - & - & - & - & + & - \\
\hline 4 & DLBCL & - & + & + & - & Non-GCB & - & - & - & - & + & - \\
\hline 5 & DLBCL & - & + & + & - & Non-GCB & - & - & + & - & + & - \\
\hline $6 a$ & DLBCL & - & + & + & - & Non-GCB & $1+$ & - & - & - & - & - \\
\hline $6 b$ & DLBCL & - & + & + & - & Non-GCB & - & - & - & - & - & - \\
\hline 7 & DLBCL & - & + & + & - & Non-GCB & - & - & + & - & + & - \\
\hline 8 & DLBCL & - & + & + & - & Non-GCB & $2+$ & ND & ND & ND & ND & ND \\
\hline 9 & DLBCL & - & ND & ND & ND & Undetermined & ND & ND & ND & ND & ND & ND \\
\hline
\end{tabular}

DLBCL, diffuse large B-cell lymphoma; GCB, germinal center B-cell phenotype; ND, not done due to lack of tissue; PBL, plasmablastic lymphoma.

ally rearranged T-cell receptor genes, but unfortunately the DNA obtained from paraffin sections was too poor for analysis (only $100 \mathrm{bp}$ PCR products amplifiable using the BIOMED-2 specimen control reaction (InVivoScribe Technologies, La Ciotat, France).

\section{In Situ Hybridization}

The tumor cells of both Case 1 (plasmablastic lymphoma) and Case 10 (extranodal NK/T-cell lymphoma, nasal type) were diffusely positive for EBER (grade $4+$ ). The extent of EBER positivity in Case $6 \mathrm{a}$ and Case 8 was grades $1+$ and $2+$, respectively. The other remaining tumors, including Case 6b (a relapsed tumor in the nasal cavity), were negative for EBER.

\section{FISH Analysis}

FISH study was performed in the first seven cases of B-cell lymphoma, as there was no tissue left after immunophenotyping of Cases 8 and 9. The results are listed in Table 2. The tumor cells of the first case, a plasmablastic lymphoma, were negative for rearrangements in all the $M Y C, I G H, B C L 2, B C L 6$, and CCND1 loci investigated. Of the 6 diffuse large B-cell lymphomas, 5 (83\%) showed BCL6 gene translocation, and 2 of these 5 cases exhibited an additional $I G H$ rearrangement (Cases 5 and 7). None of the six diffuse large B-cell lymphomas showed rearrangements in $M Y C, B C L 2$, or $C C N D 1$ loci. Both specimens of the remaining patient with diffuse large B-cell lymphoma (Case 6) were negative for rearrangement of all the investigated loci.

\section{Discussion}

Primary adrenal lymphoma is a rare disease and most of the papers in the English literature are single-case reports with very few studies comprising significant numbers of cases. According to the literature, the most common presenting symptoms are abdominal or back pain, fever of unknown origin, anorexia, weight loss, and signs of adrenal insufficiency such as hypoglycemia, hyponatremia, and Addisonian crisis. ${ }^{1,4}$ In our series of 10 patients, the most common presenting symptoms were pain, fever, or both. Only one patient presented with clinical features of adrenal insufficiency. The earlier case reports and literature reviews based mostly on these single-case reports reveal a high rate of adrenal insufficiency (67-69\%) and bilateral involvement $(60-79 \%)^{1,4}$ In our series, only 10 and $50 \%$ patients, respectively, had adrenal insufficiency and bilateral involvement. We speculate that the very high rates of adrenal insufficiency and bilateral involvement in the earlier literature reviews were the results of publication bias.

In this series of 10 cases of primary adrenal lymphoma, we found that $90 \%$ were of B-cell lineage including 1 plasmablastic lymphoma and 8 diffuse large B-cell lymphomas. The plasmablastic lymphoma comprised monotonous tumor cells expressing plasma cell-associated markers such as CD138 and downregulated CD20, an immunophenotype indicative of terminal B-cell differentiation. This patient had no risk factor for HIV and she did not have history of constitutional or iatrogenic immunosuppression or autoimmune disease. In our earlier study of 50 diffuse large B-cell lymphomas with low/absent CD20/CD79a and an immunophenotype indicative of terminal B-cell differentiation (MUM1/CD38/CD138/EMA-positive), we were able to define several distinct subgroups including $23(46 \%)$ cases of plasmablastic lymphoma of the oral mucosa type and 17 (34\%) cases of plasmablastic lymphoma with plasmacytic differentiation. ${ }^{10}$ The former group showed a monomorphic population of immunoblasts with no or minimal plasmacytic differentiation and most patients were HIV +, 74\% tumors were EBV-positive, and nearly half of cases presented in the oral cavity. In the latter group, only $33 \%$ were HIV +, EBV was 

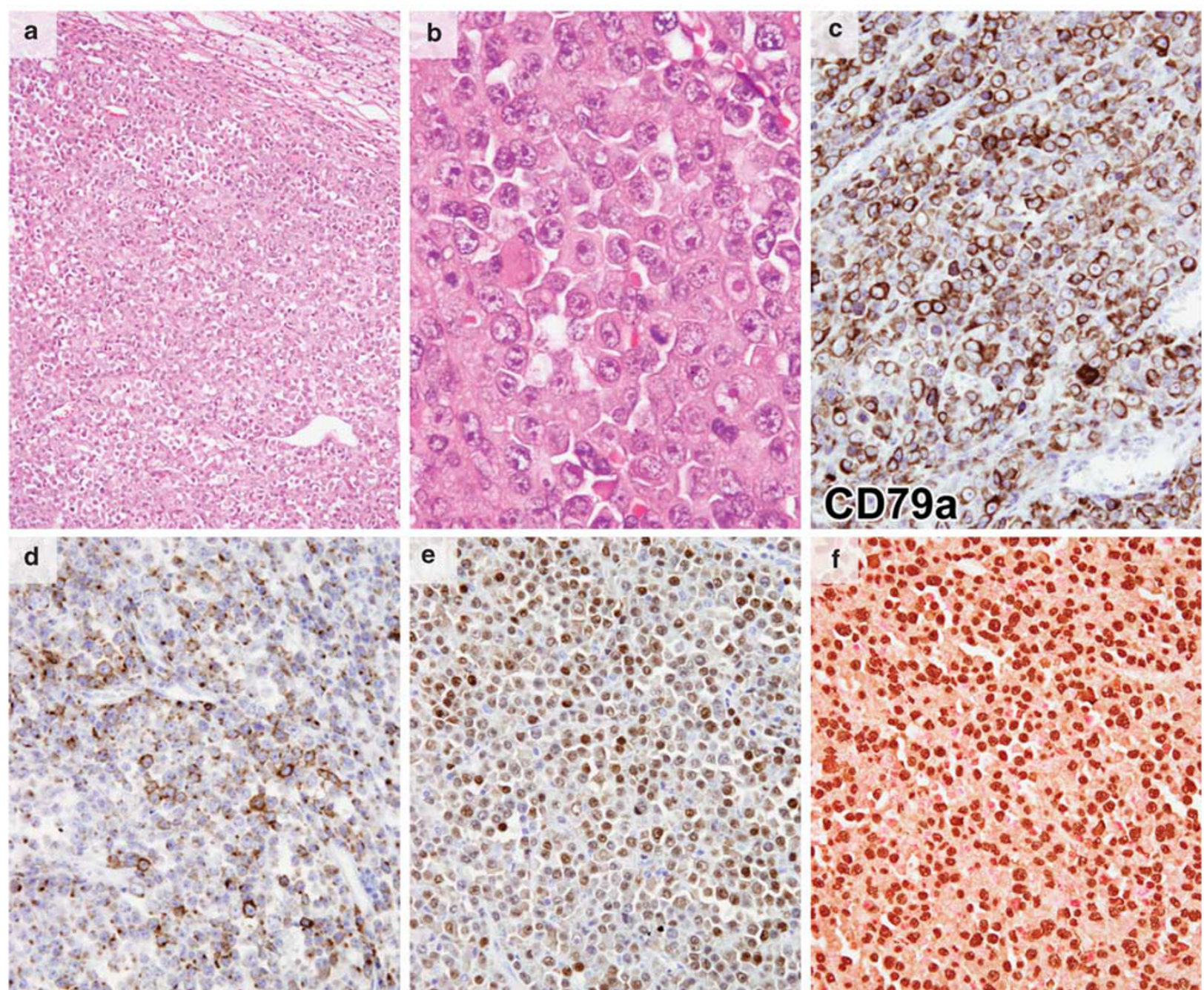

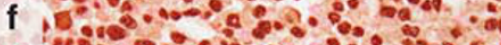

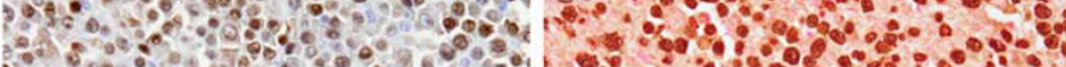

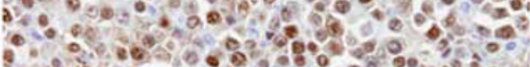
$400.05000 \%$ $3: 030$.

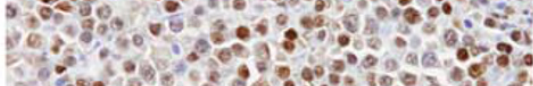

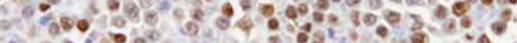

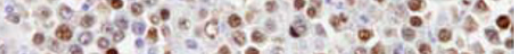
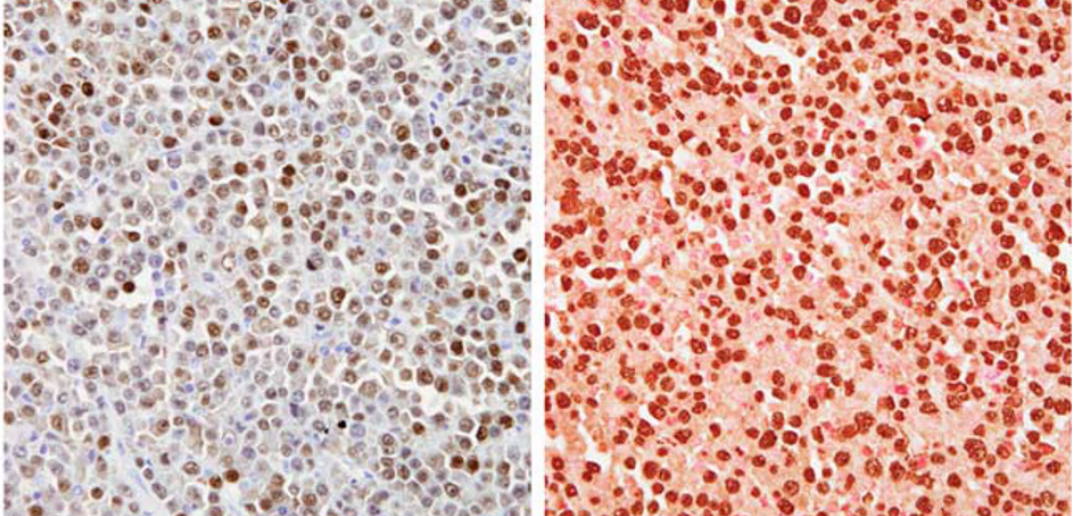

\section{CD138 21093}

MUM1 $3 \%: \%$
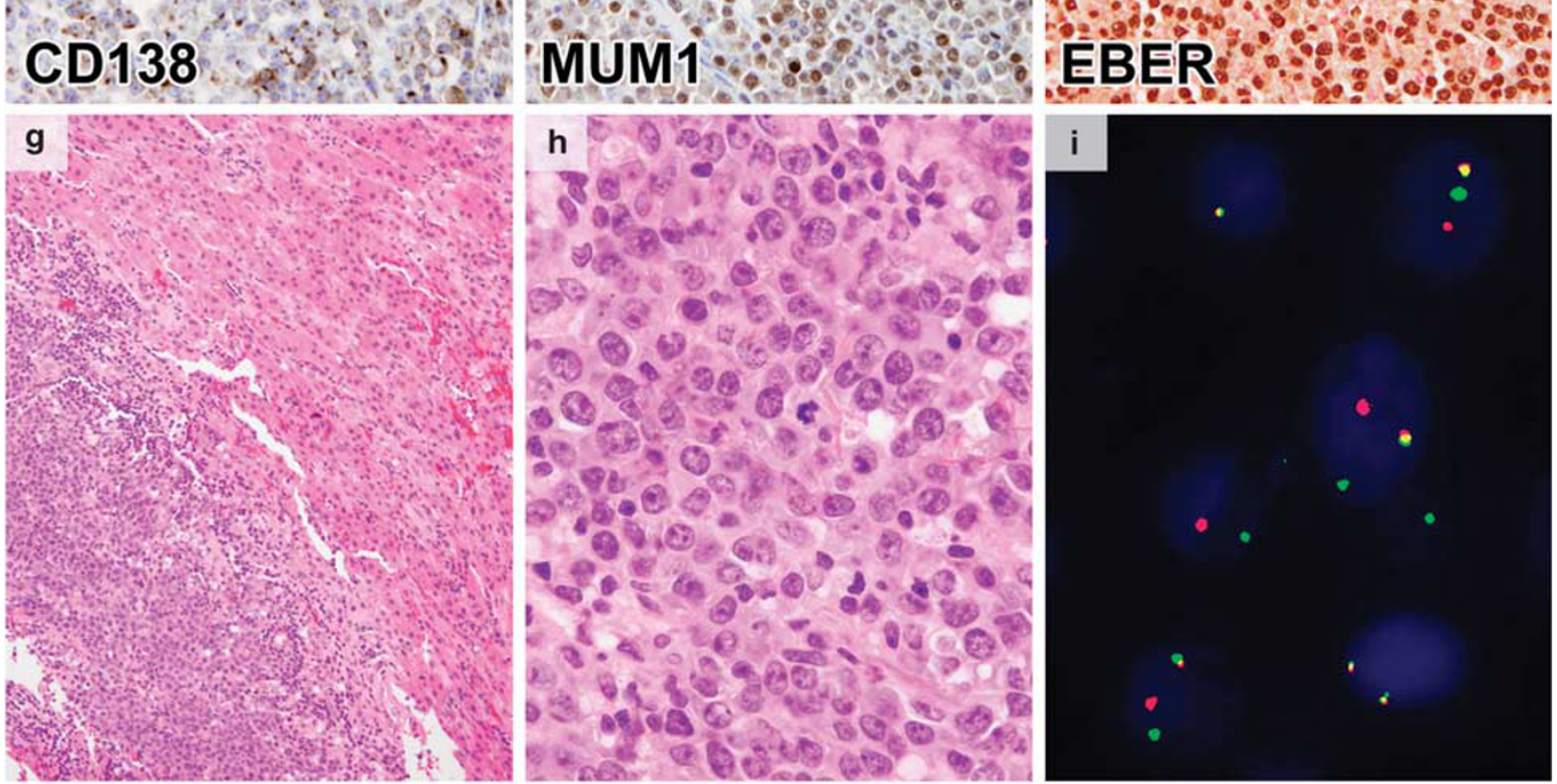

Figure 1 Photomicrographs of Case 1 (PBL, panels a-f) and Case 5 (DLBCL, panels g-i). In Case 1, medium-power view shows diffuse tumor infiltration with residual adrenal cortex at the upper field (a, H\&E stain $\times 200$ ), whereas high-power shows large plasmablasts with a prominent nucleolus and abundant cytoplasm $(\mathbf{b}$, H\&E stain $\times 1000)$. The tumor cells express CD79a $(\mathbf{c}$, $\times 400)$, CD138 (hererogeneous, $\mathbf{d} \times 400)$, and MUM1 $(\mathbf{e}, \times 400)$, and are diffusely positive for EBER $(\mathbf{f}, \times 400)$. Case 5 is a DLBCL with diffuse infiltration of tumor cells $(\mathbf{g}, \times 200)$ with adrenal cortex in the upper field. The tumor cells are large with centroblastic appearance (h, $\times 400)$. FISH shows BCL6 gene translocation with dual-color break-apart probes (i). 

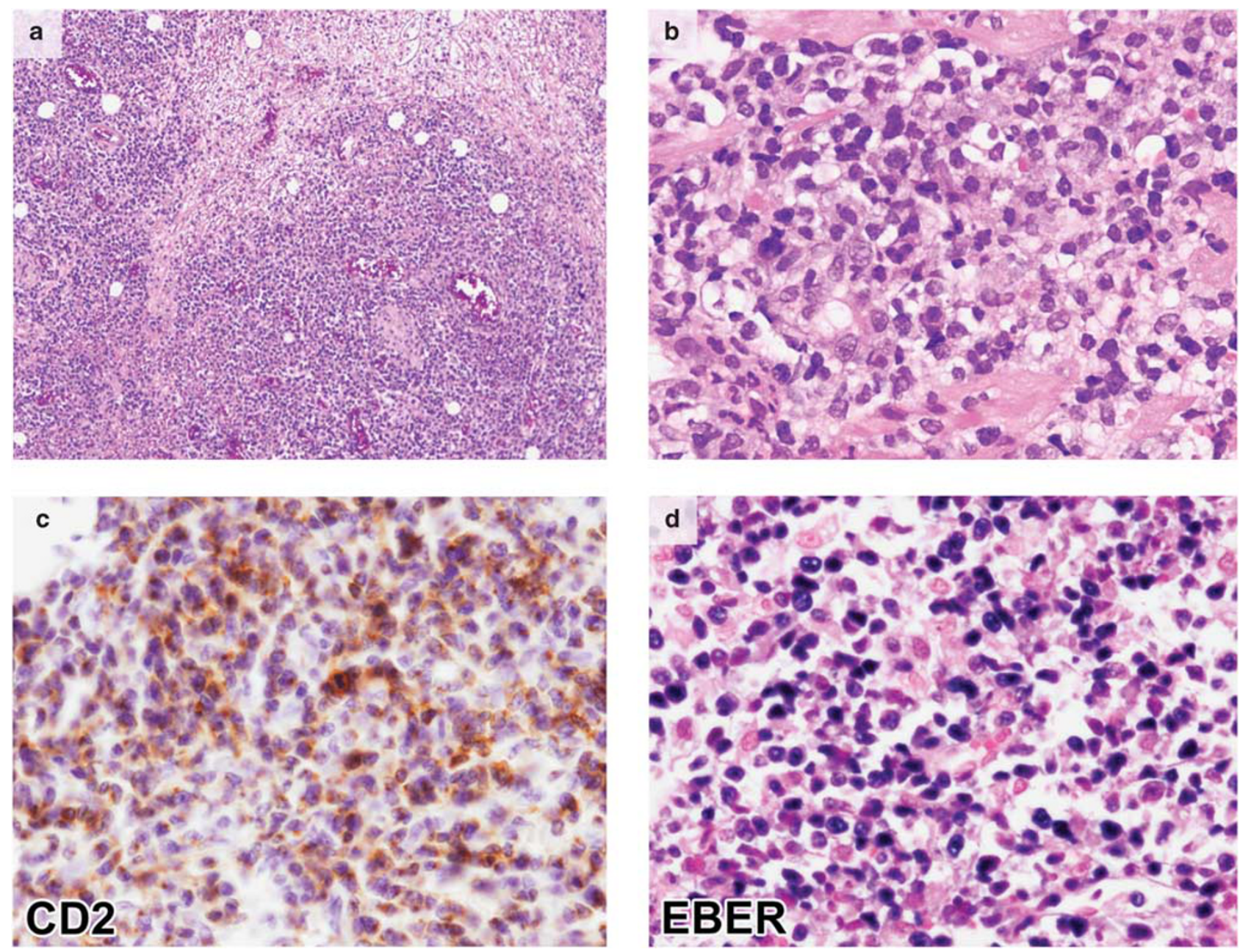

Figure 2 Photomicrographs of Case 10, NK/T-cell lymphoma. Low-power view shows an extensive infiltration by neoplastic cells (a, original magnification, $\times 200$ ). High-power view shows a tumor composed mainly of medium-sized cells with irregular nuclei (b) original magnification, $\times 600$ ). The tumor cells express CD2 (c) and are positive for EBER (d) (both original magnification, $\times 400$ ).

detected in $62 \%$, and $44 \%$ had nodal presentation. The tumors of both subgroups were aggressive. Case 1 in the present series, HIV-negative and EBVpositive with a poor prognosis, belonged to the latter subgroup.

Gene expression profiling studies of diffuse large B-cell lymphoma have identified two major subtypes, those with an expression pattern similar to normal germinal center B-cells and those similar to the activated peripheral blood cells, with the former carrying a significantly better prognosis after chemotherapy. ${ }^{11}$ Using immunohistochemistry (CD10, bcl-6, and IRF4/MUM-1) Hans et $a l^{9}$ classified diffuse large B-cell lymphomas into germinal center B-cell vs non-germinal center B-cell phenotype and found a good concordance with gene expressing profiling and a better prognosis in the group with germinal center B-cell phenotype than that with non-germinal center B-cell phenotype, a similar finding to the gene expression profiling studies. Following the Hans algorithm, we found that all diffuse large B-cell lymphomas in our study were of a non-germinal center B-cell phenotype, and such phenotype might be one of the reasons for the poor prognosis of our patients.

BCL6 gene rearrangement is the most common chromosomal translocation identified in diffuse large B-cell lymphoma, occurring in up to $30 \%$ cases albeit with a lower incidence in Taiwanese patients $(17 \%))^{12-15}$ It is more frequently detected in diffuse large B-cell lymphomas with an activated Bcell/non-germinal center B-cell phenotype than in those with a germinal center B-cell phenotype. ${ }^{16,17}$ The significance of BCL6 gene rearrangement in disease presentation and survival is controversial. Using Southern blot analysis, Offit et $a l^{13}$ found that $23 \%(23 / 102)$ of diffuse large B-cell lymphomas exhibited BCL6 rearrangement and this rearrangement correlated with a favorable clinical outcome. This effect might have been due to an association of $B C L 6$ rearrangements with other cytogenetic indicators of progression [(trisomy 7, trisomy 12, $\operatorname{del}(6)(q 21 \mathrm{q} 25)] .{ }^{14}$ On the other hand, neither Bastard et $a l^{12}$ nor Iqbal et $a l^{17}$ found an association 
Table 3 Laboratory findings of three primary adrenal NK/T-cell lymphomas

\begin{tabular}{lcccccccccccccccc}
\hline & CD2 & CD3 & CD5 & CD7 & CD8 & CD56 & TIA-1 & Gr B & BF1 & LMP-1 & EBER & TCR & \\
\hline Mizoguchi et al l $^{20}$ & NA & - & - & NA & NA & + & + & + & NA & NA & + & Germline \\
Thompson et al & + & + & - & - & - & + & + & + & NA & NA & + & ND \\
Case 10 of this report & + & + & - & + & + & + & + & + & + & - & + & ND (poor DNA)
\end{tabular}

NA, not available; ND, not done.

between BCL6 rearrangement and initial features of the disease or clinical outcome. In a large series of de novo diffuse large B-cell lymphomas, Kramer et al found that BCL6 rearrangement was more frequent in patients with extranodal (36\%) and extensive $(39 \%)$ presentation vs primary nodal disease $(28 \%)$. However, there was no significant correlation with disease stage, lymphadenopathy, bone marrow involvement, disease-free survival, or overall survival. ${ }^{18}$ In Taiwanese patients with diffuse large B-cell lymphomas, Chen et $a l^{15}$ found no association between BCL6 gene alternations and clinical characteristics (including nodal vs extranodal tumors) or prognosis, probably because the case number with BCL6 gene rearrangement was small (10 of 59 cases). In contrast to these studies, Barrans et $a l^{16}$ identified rearrangements of the BCL6 locus in $25 \%$ diffuse large B-cell lymphomas and found this to be associated with a poor prognosis. In our series of primary adrenal lymphoma, although the case number was small, we have observed a high frequency of $B C L 6$ rearrangement in diffuse large Bcell lymphoma patients $(5 / 6$ or $83 \%)$ and a poor prognosis. Of note, $4(80 \%)$ of 5 Taiwanese diffuse large B-cell lymphomas carried BCL6 rearrangement, which is high as compared with the earlier reported $17 \%$. Further studies of larger series of cases are needed to establish the prognostic significance of $B C L 6$ translocation on primary adrenal lymphoma of the diffuse large B-cell lymphomas subtype.

Extranodal NK/T-cell lymphoma, nasal type is a predominately extranodal lymphoma with a cytotoxic phenotype and a strong association with EBV. The upper aerodigestive tract is most commonly involved with other preferential sites including the skin, soft tissue, gastrointestinal tract, and testis. ${ }^{19}$ To date, only two cases of primary adrenal NK/T lymphoma have been described in the English literature. The first was a 17-year-old boy with fever and bilateral adrenal tumors who died in 4 days from EBV-related hemophagocytic lymphohistiocytosis with multiple organ failure resulting from a cytokine storm (hypercytokinemia). ${ }^{20}$ The second patient was a 37-year-old male with a homogeneous enlargement of the left adrenal gland and a rapid recurrence in the contralateral gland after surgical excision who died of disease progression in 4 months. ${ }^{21}$ Our Case 10 is the third case, and the pertinent laboratory findings of these cases are summarized in Table 3. All three tumors expressed
CD56, TIA-1, and granzyme B and were positive for EBER. The clinical course was very aggressive, similar to extranodal NK/T-cell lymphomas, nasal type at other anatomical sites. ${ }^{19}$ The great majority of such tumors are of NK-cell lineage, although some are of cytotoxic T-cell phenotype. Interestingly, the tumor cells of our case expressed $\beta \mathrm{F} 1$, indicating a possible cytotoxic T-cell origin. Recently, Park et $a l^{22}$ described a series of systemic EBV-positive T-cell lymphoma in immunocompetent elderly patients, which they proposed as a distinct entity related to an underlying dysfunction of the T-cell immunity resulting in a failure to eradicate the EBV infection. These patients usually presented with generalized lymphadenopathy, in contrast to our Case 10 who presented with a localized disease.

In summary, we have presented the detailed clinicopathological features of 10 cases of primary adrenal lymphoma including one case each of plasmablastic lymphoma and extranodal NK/T-cell lymphoma, nasal type. The majority are diffuse large B-cell lymphomas, which have a non-germinal center B-cell phenotype and a high frequency of $B C L-6$ rearrangement. These tumors are aggressive as reported earlier., ${ }^{2,3}$ Although all our cases presented with a localized disease, the prognosis of the entire group was worse as compared with that of their nodal counterparts or that of diffuse large Bcell lymphomas at most other extranodal sites. ${ }^{23-25}$ The reasons for the aggressive behavior are unknown and may be attributable to bulky tumor size at presentation, non-germinal center B-cell phenotype, and $B C L-6$ gene rearrangement in the diffuse large B-cell lymphoma cases.

\section{Acknowledgement}

This work was supported by research grants from Chi-Mei Medical Center, Tainan (CMNSC9703) and National Science Council, Taipei (97-2320-B-001MY3), Taiwan, and the Association of International Cancer Research and Leukaemia Research Fund, UK. CMB is funded by a Senior Clinician Scientist Fellowship from The Health Foundation/The Royal College of Pathologists/The Pathological Society of Great Britain and Ireland. AM is supported by a research contract from the Hospital Clinic and EC is funded by the Spanish Ministry of Science and Innovation SAF08-3630. 


\section{Conflict of interest}

The authors declare no conflict of interest.

\section{References}

1 Singh D, Kumar L, Sharma A, et al. Adrenal involvement in non-Hodgkin's lymphoma: four cases and review of literature. Leuk Lymphoma 2004;45:789-794.

2 Ohsawa M, Tomita Y, Hashimoto M, et al. Malignant lymphoma of the adrenal gland: its possible correlation with the Epstein-Barr virus. Mod Pathol 1996;9: 534-543.

3 Grigg AP, Connors JM. Primary adrenal lymphoma. Clin Lymphoma 2003;4:154-160.

4 Mantzios G, Tsirigotis P, Veliou F, et al. Primary adrenal lymphoma presenting as Addison's disease: case report and review of the literature. Ann Hematol 2004;83:460-463.

5 Schreiber CS, Sakon JR, Simiao FP, et al. Primary adrenal lymphoma: a case series study. Ann Hematol 2008;87:859-861.

6 Ide M, Fukushima N, Hisatomi T, et al. Non-germinal cell phenotype and bcl-2 expression in primary adrenal diffuse large B-cell lymphoma. Leuk Lymphoma 2007;48:2244-2246.

7 Chuang SS, Ye H, Du MQ, et al. Histopathology and immunohistochemistry in distinguishing Burkitt lymphoma from diffuse large B-cell lymphoma with very high proliferation index and with or without a starrysky pattern: a comparative study with EBER and FISH. Am J Clin Pathol 2007;128:558-564.

8 Martin-Subero JI, Chudoba I, Harder L, et al. Multicolor-FICTION: expanding the possibilities of combined morphologic, immunophenotypic, and genetic single cell analyses. Am J Pathol 2002;161: 413-420.

9 Hans CP, Weisenburger DD, Greiner TC, et al. Confirmation of the molecular classification of diffuse large B-cell lymphoma by immunohistochemistry using a tissue microarray. Blood 2004;103:275-282.

10 Colomo L, Loong F, Rives S, et al. Diffuse large B-cell lymphomas with plasmablastic differentiation represent a heterogeneous group of disease entities. Am J Surg Pathol 2004;28:736-747.

11 Rosenwald A, Wright G, Chan WC, et al. The use of molecular profiling to predict survival after chemotherapy for diffuse large-B-cell lymphoma. N Engl J Med 2002;346:1937-1947.

12 Bastard C, Deweindt C, Kerckaert JP, et al. LAZ3 rearrangements in non-Hodgkin's lymphoma: correla- tion with histology, immunophenotype, karyotype, and clinical outcome in 217 patients. Blood 1994;83:2423-2427.

13 Offit K, Lo Coco F, Louie DC, et al. Rearrangement of the bcl-6 gene as a prognostic marker in diffuse largecell lymphoma. N Engl J Med 1994;331:74-80.

14 Offit K, Louie DC, Parsa NZ, et al. BCL6 gene rearrangement and other cytogenetic abnormalities in diffuse large cell lymphoma. Leuk Lymphoma 1995;20:85-89.

15 Chen PM, Yang MH, Yu IT, et al. Low incidence of BCL-6 gene alterations for diffuse large B-cell lymphomas in Taiwan Chinese. Cancer 2002;94: 2635-2644.

16 Barrans SL, O’Connor SJ, Evans PA, et al. Rearrangement of the BCL6 locus at 3q27 is an independent poor prognostic factor in nodal diffuse large B-cell lymphoma. Br J Haematol 2002;117:322-332.

17 Iqbal J, Greiner TC, Patel K, et al. Distinctive patterns of BCL6 molecular alterations and their functional consequences in different subgroups of diffuse large Bcell lymphoma. Leukemia 2007;21:2332-2343.

18 Kramer $\mathrm{MH}$, Hermans J, Wijburg E, et al. Clinical relevance of BCL2, BCL6, and MYC rearrangements in diffuse large B-cell lymphoma. Blood 1998;92: 3152-3162.

19 Chan JK, Sin VC, Wong KF, et al. Nonnasal lymphoma expressing the natural killer cell marker CD56: a clinicopathologic study of 49 cases of an uncommon aggressive neoplasm. Blood 1997;89:4501-4513.

20 Mizoguchi Y, Nakamura K, Miyagawa S, et al. A case of adolescent primary adrenal natural killer cell lymphoma. Int J Hematol 2005;81:330-334.

21 Thompson MA, Habra MA, Routbort MJ, et al. Primary adrenal natural killer/T-cell nasal type lymphoma: first case report in adults. Am J Hematol 2007;82: 299-303.

22 Park S, Kim K, Kim WS, et al. Systemic EBV+ T-cell lymphoma in elderly patients: comparison with children and young adult patients. Virchows Arch 2008;453:155-163.

23 Hsu CW, Ho CL, Sheu WH, et al. Adrenal insufficiency caused by primary aggressive non-Hodgkin's lymphoma of bilateral adrenal glands: report of a case and literature review. Ann Hematol 1999;78:151-154.

24 Ha CS, Dubey P, Goyal LK, et al. Localized primary non-Hodgkin lymphoma of the breast. Am J Clin Oncol 1998;21:376-380.

25 Nakamura S, Matsumoto T, Iida M, et al. Primary gastrointestinal lymphoma in Japan: a clinicopathologic analysis of 455 patients with special reference to its time trends. Cancer 2003;97:2462-2473. 